\title{
Development and evaluation of a new Plasmodium falciparum 3D7 blood stage malaria cell bank for use in malaria volunteer infection studies
}

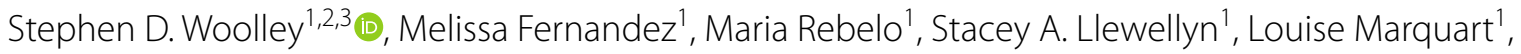 \\ Fiona H. Amante ${ }^{1}$, Helen E. Jennings ${ }^{1}$, Rebecca Webster ${ }^{1}$, Katharine Trenholme ${ }^{1,4}$, Stephan Chalon ${ }^{5}$, \\ Joerg J. Moehrle ${ }^{5}$, James S. McCarthy ${ }^{1}$ and Bridget E. Barber ${ }^{1 *}$
}

\begin{abstract}
Background: New anti-malarial therapeutics are required to counter the threat of increasing drug resistance. Malaria volunteer infection studies (VIS), particularly the induced blood stage malaria (IBSM) model, play a key role in accelerating anti-malarial drug development. Supply of the reference 3D7-V2 Plasmodium falciparum malaria cell bank (MCB) is limited. This study aimed to develop a new MCB, and compare the safety and infectivity of this MCB with the existing 3D7-V2 MCB, in a VIS. A second bank (3D7-V1) developed in 1995 was also evaluated.

Methods: The 3D7-V2 MCB was expanded in vitro using a bioreactor to produce a new MCB designated 3D7-MBE-008. This bank and 3D7-V1 were then evaluated using the IBSM model, where healthy participants were intravenously inoculated with blood-stage parasites. Participants were treated with artemether-lumefantrine when parasitaemia or clinical thresholds were reached. Safety, infectivity and parasite growth and clearance were evaluated.

Results: The in vitro expansion of 3D7-V2 produced 200 vials of the 3D7-MBE-008 MCB, with a parasitaemia of 4.3\%. This compares to $0.1 \%$ in the existing 3D7-V2 MCB, and $<0.01 \%$ in the 3D7-V1 MCB. All four participants (two per MCB) developed detectable $P$. falciparum infection after inoculation with approximately 2800 parasites. For the 3D7-MBE-008 MCB, the parasite multiplication rate of $48 \mathrm{~h}\left(\mathrm{PMR}_{48}\right)$ using non-linear mixed effects modelling was 34.6 (95\% Cl 18.5-64.6), similar to the parental 3D7-V2 line; parasitaemia in both participants exceeded 10,000/mL by day 8. Growth of the 3D7-V1 was slower ( $\mathrm{PMR}_{48}$ of 11.5 [95\% Cl 8.5-15.6]), with parasitaemia exceeding 10,000 parasites/ $\mathrm{mL}$ on days 10 and 8.5. Rapid parasite clearance followed artemether-lumefantrine treatment in all four participants, with clearance half-lives of 4.01 and 4.06 (weighted mean 4.04 [95\% Cl 3.61-4.57]) hours for 3D7-MBE-008 and 4.11 and 4.52 (weighted mean 4.31 [95\% Cl 4.16-4.47]) hours for 3D7-V1. A total of 59 adverse events occurred; most were of mild severity with three being severe in the 3D7-MBE-008 study.
\end{abstract}

Conclusion: The safety, growth and clearance profiles of the expanded 3D7-MBE-008 MCB closely resemble that of its parent, indicating its suitability for future studies. Trial Registration: Australian New Zealand Clinical Trials registry numbers: P3487 (3D7-V1): ACTRN12619001085167. P3491 (3D7-MBE-008): ACTRN12619001079134

Keywords: Plasmodium falciparum, Induced blood-stage malaria, CHMI, VIS, Malaria

\footnotetext{
${ }^{*}$ Correspondence: bridget.barber@qimrberghofer.edu.au

${ }^{1}$ QIMR Berghofer Medical Research Institute, Brisbane, QLD, Australia

Full list of author information is available at the end of the article
}

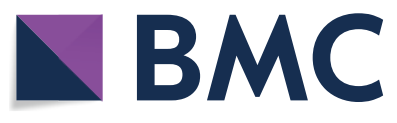

(c) The Author(s) 2021. This article is licensed under a Creative Commons Attribution 4.0 International License, which permits use, sharing, adaptation, distribution and reproduction in any medium or format, as long as you give appropriate credit to the original author(s) and the source, provide a link to the Creative Commons licence, and indicate if changes were made. The images or other third party material in this article are included in the article's Creative Commons licence, unless indicated otherwise in a credit line to the material. If material is not included in the article's Creative Commons licence and your intended use is not permitted by statutory regulation or exceeds the permitted use, you will need to obtain permission directly from the copyright holder. To view a copy of this licence, visit http://creativeco mmons.org/licenses/by/4.0/. The Creative Commons Public Domain Dedication waiver (http://creativecommons.org/publicdomain/ zero/1.0/) applies to the data made available in this article, unless otherwise stated in a credit line to the data. 


\section{Background}

Malaria continues to cause major morbidity and mortality worldwide, with current control measures being threatened by the spread of artemisinin-resistance in the Greater Mekong Subregion [1-3]. New anti-malarial drugs and vaccines are, therefore, urgently required. The current anti-malarial drug pipeline has been accelerated by the use of human volunteer infection studies (VIS) [4-8], particularly the induced blood-stage malaria model (IBSM) $[5,6]$. In these studies, healthy, malaria-naïve participants are inoculated with Plasmodium-infected erythrocytes, enabling the assessment of the blood stage schizont activity of antimalarial drug candidates [5, 6, 9-11]. As of March 2020, 401 volunteers have been inoculated with the Plasmodium falciparum 3D7 clone, most at QIMR Berghofer in Brisbane, Australia $(\mathrm{n}=335)$, but some at sites in the Netherlands and UK $(n=66)[6,12-16]$.

The current QIMR Berghofer malaria cell bank (MCB) used to inoculate volunteers with $P$. falciparum, termed 3D7-V2, was produced in 1995 [17-19]. At that time, two volunteers were experimentally infected by mosquito bite with Plasmodium falciparum 3D7, and $500 \mathrm{~mL}$ of blood was collected from each volunteer six hours following the onset of fever [19]. Although two MCBs were produced (3D7-V1 and 3D7-V2), the higher parasitaemia in the 3D7-V2 bank $(0.1 \%$ compared to $<0.01 \%$ of erythrocytes parasitized, respectively) has led to this bank being utilized in subsequent malaria VIS. The 3D7-V1 has been utilized only once, for re-inoculation into the original donor [19].

Stocks of the $P$. falciparum 3D7-V2 MCB are limited, therefore, further MCBs are required to ensure an ongoing supply of this valuable resource. The development of further banks can be undertaken by collection of samples from malaria-infected patients or experimentally infected volunteers [17]. An alternative approach is the in vitro manufacture of banks using a bioreactor, such as the Wave ${ }^{\mathrm{TM}} 25$ bioreactor system [17]. This method has been used previously to produce and test in vivo two cell banks, a genetically modified $P$. falciparum blood stage-cell bank [17], and an arteminisin-resistant $P$. falciparum cell bank [20]. This proved to be a cost-efficient method for the production of a MCB for use in IBSM studies [17, 21]. This method also allows for blood group selection of the MCB.

The development of a new MCB, 3D7-MBE-008 (MBE-008), using this biomanufacture process, and the clinical evaluation of this MCB is reported. Safety, infectivity and parasite growth and clearance of the 3D7-MBE-008 and the previous 3D7-V1 were compared to the existing data on the 3D7-V2 bank.

\section{Methods}

Development of 3D7-MBE-008 Master Cell Bank

The 3D7-MBE-008 MCB was manufactured in accordance with Good Manufacturing Practice standards [22] in 2015 using the previously described method [17]. In brief, a single vial of the 3D7-V2 MCB was thawed and expanded using the bioreactor. Erythrocytes used in the production of the $\mathrm{MCB}$ were from a single blood group $\mathrm{O}$ $\mathrm{Rh}$ (D) negative donor, provided by Lifeblood (formerly Australian Red Cross Blood Service). The donor was screened in accordance with TGA regulatory requirements for donation of blood for transfusion. Pooled, heat inactivated serum collected from donors by Key Biologics (Memphis, Tennessee, U.S.) used in the manufacturing process was also extensively screened. The final 3D7-MBE-008 culture was cryopreserved with Glycerolyte 57 in 1:2.2 ratio, and aliquoted to produce $2001 \mathrm{~mL}$ cryovials, which were stored between -140 and $-196{ }^{\circ} \mathrm{C}$ in secure, monitored vapour phase liquid nitrogen tanks at Q-Gen Cell Therapeutics, Brisbane, Australia.

\section{Laboratory testing of the Master Cell Banks}

The percentage of parasitized erythrocytes and the percentage of ring-stage parasites were determined via thin film microscopy for 3D7-MBE-008 and thick film microscopy for 3D7-V1. Testing for microbial contamination was performed in line with the British Pharmacopoeia Appendix XVI E- microbial contamination of cellular products [23].

Parasite viability of 3D7-MBE-008 was determined using flow cytometry as previously described [20] at the time of manufacture and then in an ongoing stability and sterility program, with testing every 12 months. The viability of the parasites in the 3D7-V1 bank was determined at the time of manufacture by limiting dilution assay followed by PCR as previously described [19].

For confirmation of parasite identity, the DNA sequence of three widely used hypervariable genes $(P$. falciparum merozoite surface protein-1 [Pf MSP-1], $P f$ MSP-2 and $P f$ glutate-rich protein [Pf GLURP]) from 3D7-MBE-008 were compared to 3D7-V2. In vitro drug sensitivity testing to nine antimalarials was also undertaken as previously described [19].

\section{Inoculum preparation}

The viability of ring stage parasites in the MBE-008 MCB was assessed by flow cytometry as previously described [20], to identify the dilution required to achieve an inoculum dose similar to 3D7-V2 MCB. To prepare the inoculum, one or more vials of the MCB were thawed, with the resulting red cell pellet washed and resuspended in $0.9 \%$ sodium chloride. The washed cell suspension was then 
diluted with $0.9 \%$ sodium chloride to achieve the target number of viable ring-stage parasites in each $2 \mathrm{ml}$ inoculum, taking into account the characteristics of each $\mathrm{MCB}$ including the percentage of parasitized erythrocytes, the percentage of ring-stage erythrocytes, and parasite viability. The number of parasites in the final inoculum was verified by $18 \mathrm{~S}$ quantitative PCR targeting the $P$. falciparum 18S rRNA gene (qPCR) [24] with results available after inoculation.

\section{Clinical study design}

Two concurrent IBSM studies were conducted, one with the 3D7-V1 MCB and the other with 3D7-MBE-008. Each study consisted of two single-participant cohorts, with a 5 -week period between cohorts. The primary objective of both studies was safety. Secondary objectives included infectivity, parasite growth and clearance, the latter following administration of artemether-lumefantrine.

The studies were conducted at Q-Pharm Pty Ltd, Brisbane, Australia. Ethical approval was given by QIMR Berghofer Human Research Ethics Committee (HREC), and by Lifeblood HREC for P. falciparum 3D7-MBE-008. All participants gave written informed consent before enrolment. Both studies were registered with the Australian New Zealand Clinical Trials registry; 3D7-V1 (ACTRN12619001085167) and 3D7-MBE-008 (ACTRN12619001079134).

\section{Participants}

Participants were eligible if they were aged $18-55$ years, healthy and malaria-naïve (see Additional file 1: Appendix for inclusion and exclusion criteria). For the 3D7-V1 study, only males who were blood group $\mathrm{Rh}(\mathrm{D})$ positive were eligible, due to the fact that the 3D7-V1 MCB donor was $R h(D)$ positive.

\section{Procedures}

All participants were inoculated with approximately 2800 viable infected erythrocytes on Day 0. Parasitaemia was monitored by $18 \mathrm{~S}$ qPCR daily [24] from Day 4 until parasites were detected, then twice daily until artemetherlumefantrine (20 mg artemether/ $120 \mathrm{mg}$ lumefantrine; Novartis Pharmaceuticals Pty Ltd) was given, and then at specified timepoints post treatment until qPCR was negative (Additional file 1: Table S1). Figure 1a illustrates the study design for 3D7-V1 MCB and Fig. 1b illustrates the study design for 3D7-MBE-008 MCB. The protocol specified that a curative course of artemether-lumefantrine, consisting of 6 doses of 4 tablets over a 60 -h period, would be given when the participants' parasitaemia exceeded 10,000 parasites/mL, or the participants' malarial clinical score was $\geq 6$ (see Additional file 1 for calculation of malaria clinical score). However, due to slower than expected parasite growth in the first subject inoculated with 3D7-V1, and lack of any clinical symptoms in the participant at a parasite count of 10,000 parasites/ $\mathrm{mL}$, the $3 \mathrm{D} 7-\mathrm{V} 1$ protocol was amended to change the parasite treatment threshold to 100,000 parasites $/ \mathrm{mL}$.

\section{Safety assessments}

Safety was evaluated by recording all adverse events as well as any abnormal laboratory results. Investigations were performed at the timepoints specified in Additional file 1: Table S1. During every outpatient visit and during confinement, a malaria clinical score for each participant was generated. A graded assessment of symptoms and laboratory results was used (see Additional file 1).

\section{Parasite growth and clearance}

The parasite multiplication rate of $48 \mathrm{~h}\left(\mathrm{PMR}_{48}\right)$ for each $\mathrm{MCB}$ was calculated by applying the pre-treatment $\mathrm{qPCR}$ data to a sine-wave growth model, estimated using a non-linear mixed effects model in R Statistical package 3.6.1, as previously reported [25]. The $\mathrm{PMR}_{48}$ for each MCB was then presented as an estimate with a 95\% CI. To determine if there were any significant differences between the growth model parameter estimates from the new MCBs and the previously used 3D7-V2 MCB, an omnibus test for between-group differences was used [26]. The sine- wave growth model estimated using a nonlinear regression model was also used to retrospectively calculate the parasite growth characteristics of 3D7-V1 in the initial volunteer re-infected with his isolate (3D7-V1) in 1995. The parasite clearance profiles post artemetherlumefantrine treatment for each participant were estimated from the slope of best fit of the parasite clearance rate and transformed to estimate the parasite reduction ratio (PRR) per $48 \mathrm{~h}$ in the logarithmic-scale $\left(\log _{10} \mathrm{PRR}_{48}\right)$ and the parasite clearance half-life as previously reported [27], using R Statistical package 3.6.1. Parasite clearance parameters for each bank are summarized as a weighted mean and corresponding 95\% CI estimated using the inverse variance method as detailed in [27].

\section{Results \\ MCB characteristics}

The blood used for the biomanufacture of 3D7-MBE-008 tested negative for microbial contamination and for serologic evidence of infective agents. Manufacture was completed in November 2015 and produced 200 vials. The analysis of the three genetic markers ( $P f m s p-1, P f$ $m s p-2$ and Pf glurp) showed that no changes had taken place between the starting 3D7-V2 MCB and resulting 3D7-MBE-008 MCB, ruling out high level genetic change during the biomanufacturing process. The in vitro drug sensitivity of the 3D7-MBE-008 MCB showed the same 


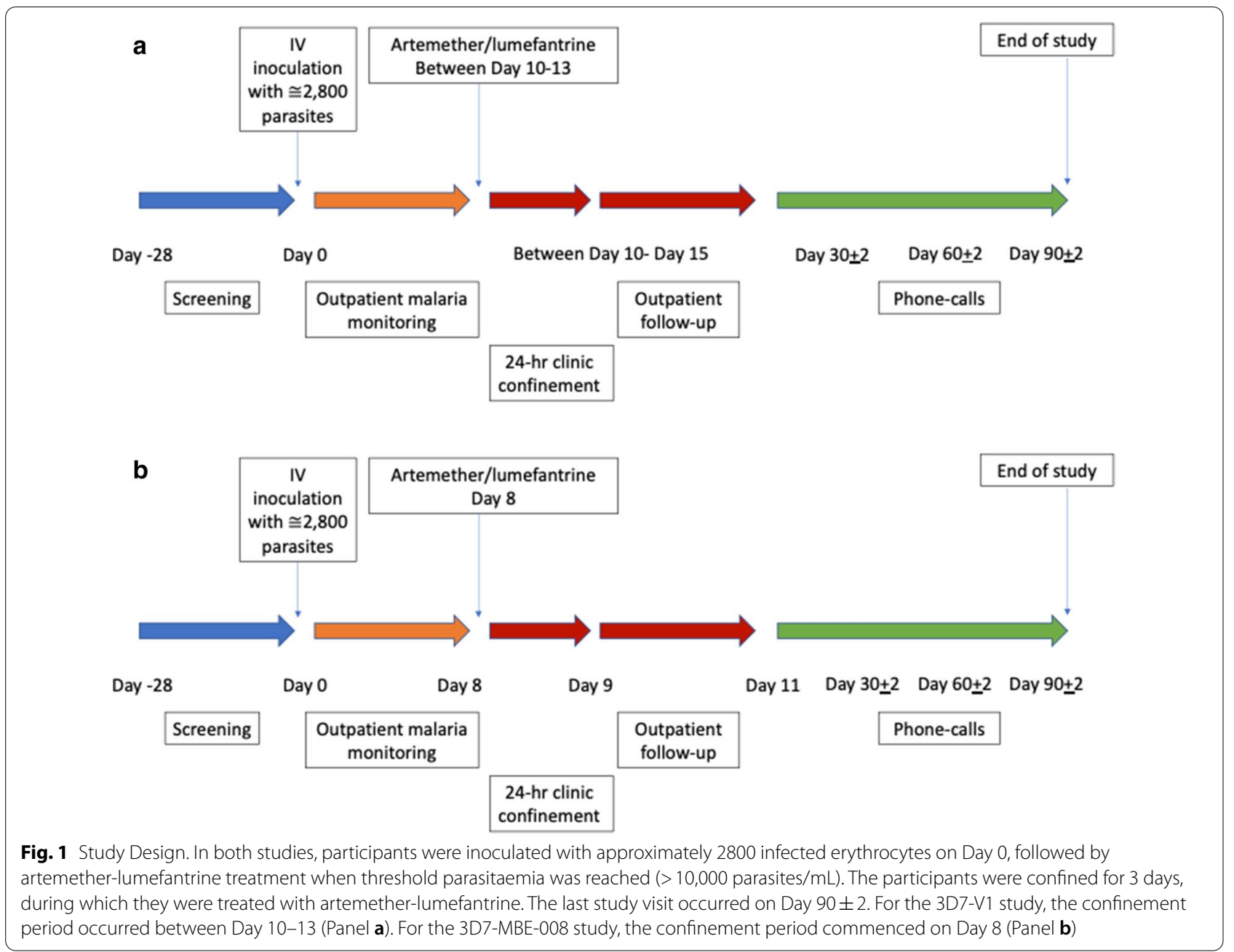

drug sensitivity profile as the established parental line to nine antimalarials (sensitive to amodiaquine, atovaquone, artemisinin, chloroquine, lumefantrine, piperaquine, pyronaridine and quinine; resistant to mefloquine). The parasite concentration of 3D7-MBE-008 MCB was 4.3\%, with $96 \%$ of parasites in ring-stage.

Viability of the 3D7-MBE-008 MCB at 12, 24 and 36 months (prior to use) was $83 \%, 71 \%$ and $63 \%$, respectively. Microbial contamination testing at these time points was negative. The parasitaemia of $3 \mathrm{D} 7-\mathrm{V} 1$ at the time of collection was $0.01 \%$. The viability as measured by limit dilution and PCR [19] at the time of manufacture was approximately $34 \%$.

\section{Study participants}

The studies were conducted between August 2019 and December 2019.Two participants were enrolled into each study. A total of 22 potential volunteers were screened for the 3D7-MBE-008 study and 19 for 3D7-V1. All four inoculated participants completed the study and are included in the safety and parasite profile analysis. The participants for the 3D7-MBE-008 study were a 32-yearold white male and a 31-year-old white non-pregnant female. The participants for the 3D7-V1 study were both 19-year-old white males.

\section{Inoculation and parasite growth}

In the 3D7-MBE-008 study, the number of parasites in each of the inocula, determined retrospectively by $18 \mathrm{~S}$ qPCR, was 18,700 and 23,100 . Both participants had parasitaemia detectable by $18 \mathrm{~S}$ qPCR on Day 4 and reached threshold parasitaemia ( $>10,000$ parasites/ $\mathrm{mL}$ ) on Day 8, when artemether-lumefantrine was commenced. The parasite counts prior to treatment were 141,416 parasites $/ \mathrm{mL}$ and 480,871 parasites $/ \mathrm{mL}$, with peak parasitaemia reached for both subjects at $2 \mathrm{~h}$ post administration of artemether-lumefantrine (239,278 parasites $/ \mathrm{mL}$ and 563,886 parasites $/ \mathrm{mL}$ ) (Fig. 2). Using the non-linear mixed effects model estimates, the estimated $\mathrm{PMR}_{48}$ was 34.6 (95\% CI 18.5-64.6) (Table 1), similar to 


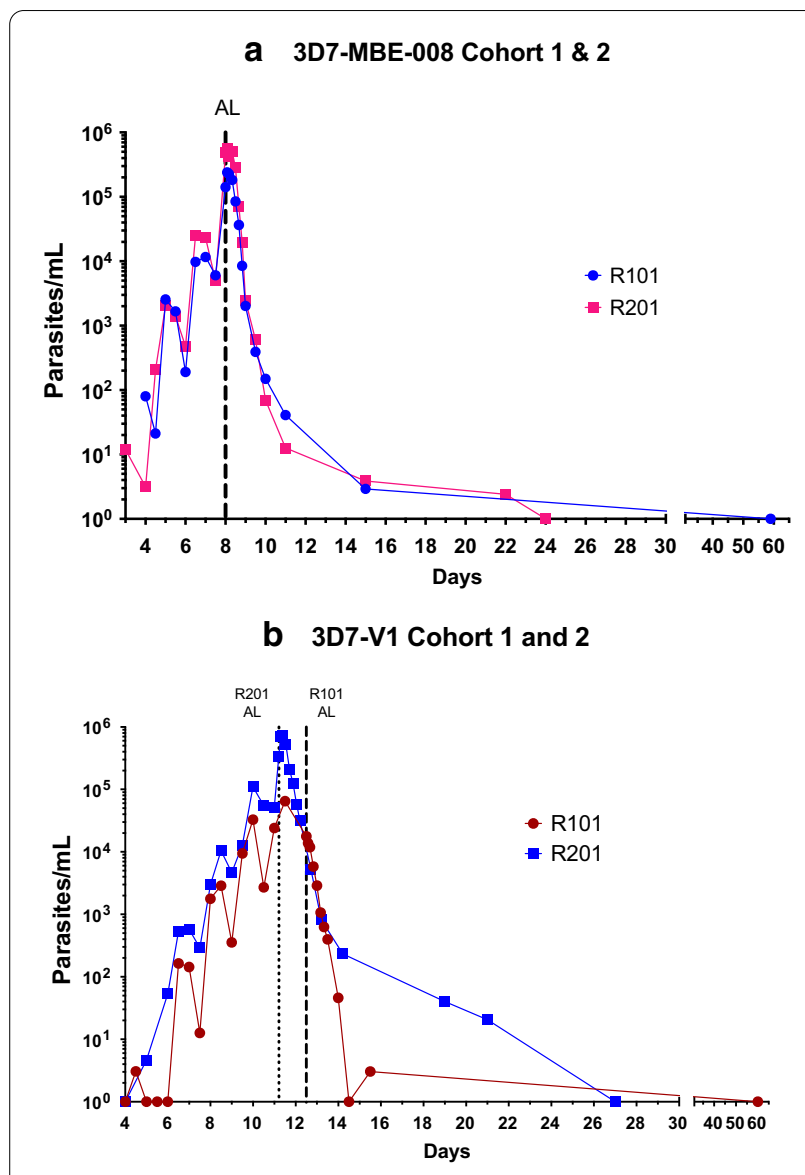

Fig. 2 Parasite growth and clearance after artemether-lumefantrine (AL) treatment Parasitaemia from Day 4 to Day 60 for the 3D7-MBE-008 study (Panel a). Parasitaemia from Day 4 to Day 60 for the 3D7-V1 study (Panel b). The vertical dashed lines represent the administration of artemether-lumefantrine treatment

that reported in a large meta-analysis of the growth rate of 3D7-V2 in previous VIS, which is 31.9 (95\% CI $28.7-$ 35.4) [25]. There were no significant differences between the growth parameters of 3D7-MBE-008 $(n=2)$ and 3D7V2 $(n=177)$ (see Additional file 1: Table S2).

In the 3D7-V1 study, the number of parasites in each inocula, determined retrospectively by qPCR, was approximately 3050 and 2694 respectively. Participant one developed detectable parasitaemia on day 5 , whereas the second participant had detectable parasitaemia on day 4 . The parasitaemia in both participants increased more slowly than those inoculated with 3D7-MBE-008, despite them being derived from the same parental 3D7 clone; on day 8 , when treatment was expected to be required, the parasitaemias of the two participants were only 1776 parasites $/ \mathrm{mL}$ and 2982 parasites $/ \mathrm{mL}$, respectively, with malaria clinical scores of zero in both participants. An urgent protocol amendment was approved by the HREC to allow treatment to be administered at a parasite threshold of $>100,000$ parasites $/ \mathrm{mL}$, and the two participants were, therefore, treated on days 12 (first participant) and day 11 (second participant). For the first participant the pre-treatment parasite count was 17,699 parasites $/ \mathrm{mL}$, and the peak parasite count (day 11) was 64,786 parasites $/ \mathrm{mL}$. For the second participant, the pretreatment parasite count was 340,789 parasites $/ \mathrm{mL}$, and the peak parasite count (occurring $4 \mathrm{~h}$ post artemetherlumefantrine) was 742,813 parasites $/ \mathrm{mL}$. The estimated $\mathrm{PMR}_{48}$ for $3 \mathrm{D} 7-\mathrm{V} 1$ was 11.5 (95\% CI 8.5-15.6) (Table 1). The primary parasitaemia data from the original 3D7V1 subject that had been calculated using a different method [19] was retrieved. Using these data in the sine wave growth model, the $\mathrm{PMR}_{48}$ for $3 \mathrm{D} 7-\mathrm{V} 1$ in the initial volunteer re-infected in 1995 was 6.4 (95\% CI 4.68.8) (Table 1). Due to the heterogeneity in the method of calculation of parasitaemia between these studies, a combined analysis was not performed. The tests of heterogeneity of the individual growth parameters of the 3D7-V1 and existing 3D7-V2 MCB showed a significant difference between the parasite growth rate $(\mathrm{p}<0.001)$

Table 1 Summary of parasite growth and clearance characteristics

\begin{tabular}{|c|c|c|c|c|}
\hline Parameter & $\begin{array}{l}\text { 3D7-V1 }(n=2) \text { Estimate } \\
(95 \% \mathrm{Cl})\end{array}$ & $\begin{array}{l}\text { 3D7-MBE-008 }(\mathrm{n}=2) \\
\text { Estimate }(95 \% \mathrm{Cl})\end{array}$ & $\begin{array}{l}\text { 3D7-V1 } 1995(\mathrm{n}=1) \\
\text { Estimate }(95 \% \mathrm{Cl})\end{array}$ & $\begin{array}{l}3 \mathrm{D} 7-\mathrm{V} 2(n=177) \\
\text { Estimate }(95 \% \mathrm{Cl}) \\
{[25]}\end{array}$ \\
\hline $\mathrm{PMR}_{48}$ & $11.5(8.5-15.6)$ & $34.6(18.5-64.6)$ & $6.4(4.6-8.8)$ & $31.9(28.7-35.4)$ \\
\hline Lifecycle (hours) & $36.5(35.0-38.0)$ & $38.8(36.7-40.8)$ & $42.5(39.2-45.8)$ & $38.8(38.3-39.2)$ \\
\hline Growth rate $\left(\log _{10}\right.$ parasites/day) & $0.52(0.30-0.74)$ & $0.80(0.54-1.10)$ & $0.35(0.13-0.58)$ & $0.63(0.59-0.66)$ \\
\hline $\mathrm{PRR}_{48}$ & $2254(1705-2979)$ & $3806(1446-10018)$ & - & - \\
\hline $\log 10 \mathrm{PRR}_{48}$ & $3.35(3.23-3.47)$ & $3.58(3.16-4.00)$ & - & - \\
\hline Parasite clearance half life & $4.31(4.16-4.47)$ & $4.04(3.61-4.57)$ & _- & _- \\
\hline
\end{tabular}

$\mathrm{n}$, number of participants; $\mathrm{Cl}, 95 \%$ confidence intervals; PMR, parasite multiplication rate; PRR, parasite reduction ratio. Growth parameter estimates are calculated from a non-linear mixed effects model. Clearance parameter estimates $\left(\mathrm{PRR}_{48}, \log _{10} \mathrm{PRR}_{48}\right.$ and parasite clearance half-life) are calculated from a weighted mean of individual clearance parameters estimates 
and parasite lifecycle $(\mathrm{p} \leq 0.001)$ (Additional file 1: Table S3).

\section{Parasite clearance}

Parasitaemia cleared in all four participants following the administration of artemether-lumefantrine. In the 3D7-MBE-008 study, the parasite clearance half-lives in the two participants were 4.01 and 4.06 (weighted mean 4.04, 95\% CI 3.61-4.57) hours, and the $\log _{10} \mathrm{PRR}_{48}{ }^{\prime} \mathrm{s}$ were 3.60 and 3.56 (weighted mean 3.58, 95\% CI 3.16-4.00) h. In the 3D7-V1 study, the parasite clearance half-lives in the two participants were 4.11 and 4.52 (weighted mean 4.31, 95\% CI 4.16-4.47) hours and the $\log _{10} \mathrm{PRR}_{48}{ }^{\prime}$ s were 3.52 and 3.20 (weighted mean 3.35, 95\% CI 3.23-3.47) h in the 3D7- V1 study (Table 1).

\section{Adverse events}

There were 35 reported adverse events in the 3D7-MBE-008 study and 24 in the 3D7-V1 study (see Table 2). In the 3D7-MBE-008 study the majority of the adverse events were mild or moderate $(32 / 35,91.4 \%)$ and attributable to early malaria $(25 / 35,71.4 \%)$; three were graded as severe (two episodes of lymphopenia; see below) and one of raised alanine transaminase [ALT]; see below). In the 3D7-V1 study, the majority of adverse events were mild $(14 / 24,58.3 \%)$, with the remainder being moderate; nearly all were attributable to early malaria $(23 / 24,95.8 \%)$. There were no serious adverse events reported in either study. The most common adverse events reported across both studies were chills $(n=6)$, headaches $(n=6)$ and myalgia $(n=7)$. One of the participants in the 3D7-MBE-008 study had a maximum malaria clinical score of 9 ( 8 h post artemether/ lumefantrine) (see Table S4). One of the participants in the 3D7-V1 study had a maximum score of 8 (36 h post artemether/lumefantrine) (see Additional file 1: Table S5).

One participant in each study developed a raised ALT. One participant in the 3D7-MBE-008 study had a peak ALT on Day 11 of 191 U/L $(4.8 \times$ ULN [upper limit of normal]) which normalized by Day 59 . The peak aspartate aminotransferase (AST), also on Day 11, was 122 U/L $(3.1 \times$ ULN $)$ and it normalized on Day 15 . The bilirubin was normal. In the 3D7-V1 study, one participant had a peak ALT on Day 14 of 128 U/L $(3.2 \times$ ULN $)$ which normalized by Day 27. The AST and bilirubin were not significantly raised.

Three participants developed transient falls in white cell counts that were classified as moderate or severe: lymphopenia (3D7-V1, nadir of $0.42 \times 10^{9} / \mathrm{L}$, lower limit of normal $\left.[\mathrm{LLN}]=1.0 \times 10^{9} / \mathrm{L}\right]$; 3D7-MBE-008, nadir of $0.34 \times 10^{9} / \mathrm{L}$ and $\left.0.39 \times 10^{9} / \mathrm{L}\right)$; neutropenia
Table 2 Adverse events reported during the studies

\begin{tabular}{|c|c|c|}
\hline Adverse event & $\begin{array}{l}\text { 3D7-V1 }(\mathrm{N}=2) \\
\mathrm{n}(\mathrm{M})\end{array}$ & $\begin{array}{l}\text { 3D7-MBE-008 } \\
(\mathrm{N}=2) n(\mathrm{M})\end{array}$ \\
\hline \multicolumn{3}{|l|}{ Systemic } \\
\hline Arthralgia & $2(2)$ & $0(0)$ \\
\hline Chills & $2(3)$ & $2(3)$ \\
\hline Decreased appetite & $1(1)$ & $1(1)$ \\
\hline Fatigue & $1(2)$ & $0(0)$ \\
\hline Feeling hot & $1(1)$ & $1(1)$ \\
\hline Headache & $2(3)$ & $2(3)$ \\
\hline Lethargy & $0(0)$ & $1(1)$ \\
\hline Malaise & $1(1)$ & $0(0)$ \\
\hline Myalgia & $2(3)$ & $2(4)$ \\
\hline Pyrexia & $2(2)$ & $2(3)$ \\
\hline Sweating & $2(2)$ & $1(3)$ \\
\hline Tachycardia & $1(1)$ & $1(2)$ \\
\hline \multicolumn{3}{|l|}{ Laboratory abnormalities } \\
\hline ALT increased & $1(1)$ & $1(1)$ \\
\hline AST increased & $0(0)$ & $1(1)$ \\
\hline Lymphocyte count decreased & $1(1)$ & $2(1)$ \\
\hline Neutrophil count decreased & $0(0)$ & $1(1)$ \\
\hline \multicolumn{3}{|l|}{ Gastrointestinal } \\
\hline Abdominal pain & $0(0)$ & $2(2)$ \\
\hline Constipation & $0(0)$ & $1(1)$ \\
\hline Diarrhoea & $0(0)$ & $2(2)$ \\
\hline \multicolumn{3}{|l|}{ Other } \\
\hline Back pain & $0(0)$ & $1(1)$ \\
\hline Erythema (from tape) & $0(0)$ & $1(1)$ \\
\hline Pain (venepuncture site) & $0(0)$ & $1(1)$ \\
\hline Ulcer (lip) & $0(0)$ & $1(1)$ \\
\hline Upper respiratory tract infection & $1(1)$ & $0(0)$ \\
\hline
\end{tabular}

$\mathrm{N}$, total number of participants in each cohort; $n$, number of participants reporting the adverse event; $M$, number of occurrences of adverse events. Adverse events were coded to System Organ Class and Preferred Term using MedDRA Version 20.1

(3D7-MBE-008 $\left.\mathrm{n}=1,1.35 \times 10^{9} / \mathrm{L}, \quad \mathrm{LLN}=1.5 \times 10^{9} / \mathrm{L}\right)$. These transient reductions were attributed to malaria.

\section{Discussion}

Here we report the manufacture and evaluation of a new P. falciparum MCB that can be utilized in future IBSM studies. In vivo testing of 3D7-MBE-008 $\mathrm{MCB}$, and the previously manufactured $3 \mathrm{D} 7-\mathrm{V} 1 \mathrm{MCB}$, indicated that they were well tolerated in healthy, malaria-naïve participants. The $\mathrm{PMR}_{48}$ for the 3D7-MBE-008 MCB was comparable to the existing 3D7-V2 MCB. In contrast, the 3D7-V1 MCB had a slower $\mathrm{PMR}_{48}$, with the parasitaemia of one participant not exceeding 10,000 parasites $/ \mathrm{mL}$ until Day 10, two days later than generally occurs with 3D7-V2. 
The parasite growth parameters of the 3D7-V1 in this study were similar to those obtained when the same nonlinear growth model was applied to the data from the initial donor re-infected with 3D7-V1 in 1995 [19, 25]. The estimated $\mathrm{PMR}_{48}$ in the two subjects in this trial was 11.5 (95\% CI 8.5-15.6) compared to 6.4 (95\% CI 4.6-8.8) in the original volunteer inoculated in 1995 . This suggests that loss of viability of the parasites after cryopreservation for over twenty years was not the reason for the slower PMR.

One possible explanation for slower than expected growth of the 3D7-V1 MCB was the lower number of infected red cells in this inoculum. Because the parasite concentrations in the individual MCBs were substantially different $(<0.01 \%$ vs $4.3 \%$ for $3 \mathrm{D} 7-\mathrm{V} 1 \mathrm{MCB}$ and 3D7-MBE-008, respectively), the 3D7-MBE-008 participants likely received a higher mean dose of erythrocytes infected with ring stage parasites. Alternatively, host factors may have played a factor. The participants in the 3D7-V1 study were younger than those in the MBE-008 study; however, analysis of data from previous studies has not found an association between age and $\mathrm{PMR}_{48}$ [25].

In addition to the slower growth rate of the 3D7-V1, another limitation for the 3D7-V1 MCB is that the original donor is $R h(D)$ positive, thus precluding $R h$ negative females from enrolling in studies using this isolate due to the risk of red cell allo-immunization. As a consequence of this and the associated slower PMR, it is likely that the 3D7-V1 MCB will be less suitable for use in further IBSM studies.

The adverse events reported for both studies are in keeping with previous IBSM studies [9-11, 28, 29]. The asymptomatic raised liver enzymes, with no associated significant rise in bilirubin, have been reported in previous IBSM studies [30, 31], sporozoite VIS [32] and in naturally occurring malaria [30,33]. Similarly, the reduction in white cell counts, especially lymphopenia and neutropenia have previously been reported in IBSM VIS, sporozoite VIS and clinical malaria [12, 16, 34-36].

Both MCBs had similar parasite clearance profiles, with no recrudescence, further confirming that both MCBs are safe to use in malaria VIS. With the development of the biomanufactured $\mathrm{MCB}$, there are now two further P. falciparum 3D7 MCBs that can be used in IBSM VIS. These are in addition to previously developed MCBs of non-falciparum species including Plasmodium vivax and Plasmodium malariae [11, 37, 38]. The development of these MCBs in falciparum and non-falciparum species may in future also enable IBSM VIS to be conducted in malaria endemic populations, to gain further understanding of host-immune response and to evaluate antimalarial drug efficacy in participants who are regularly exposed to natural malaria.
A limitation of this study was that the MCBs were each evaluated in only two participants. There is a need to, therefore, be cautious in comparing the parasite growth rates of the new MCBs against the established 3D7-V2 MCB, especially as there is variability within the observed growth rates and individual growth parameters within the 3D7-V2 MCB [25]. However, when comparing the growth rates of both 3D7-MBE-008 and 3D7-V2, the individual parameters are similar (Additional file 1: Table S2).

\section{Conclusion}

A newly developed $P$. falciparum new MCB which is safe to use in healthy, malaria-naive participants is reported. The growth characteristics of the bioreactor-expanded $P$. falciparum 3D7-MBE-008 MCB have been demonstrated and are comparable to the existing 3D7 $\mathrm{MCB}$, hence this new bank is suitable for use in future studies.

\section{Supplementary Information}

The online version contains supplementary material available at https://doi. org/10.1186/s12936-021-03627-z.

Additional file 1: Table S1. Schedule of events for both studies.

Table S2. Growth parameters of the 3D7-MBE-008 pilot bank $(n=2)$ compared to 3D7 bank using historical data $(n=177)$. Table S3. Growth parameters of the 3D7-V1 pilot bank $(n=2)$ compared to 3D7-V2 bank using historical data $(n=177)$. Table S4. Overall clinical score recorded for each participant during 3D7-MBE-008 study. Table S5. Overall clinical score recorded for each participant during 3D7-V1 study.

\section{Abbreviations}

ALT: Alanine aminotransferase; AST: Aspartate aminotransferase; BMI: Body mass index; Co-l: Co-investigator; DBP: Diastolic blood pressure; ECG: Electrocardiogram; GMP: Good Manufacturing Procedures; HBV: Hepatitis B Virus; HCV: Hepatitis C Virus; HIV: Human Immunodeficiency Virus; HR: Heart rate; HRP2: Histidine-rich protein 2; HREC: Human research ethics committee; IBSM: Induced blood-stage malaria; LDA: Limiting dilution assay; LLN: Lower limit of normal; MCB: Malaria Cell Bank; MMV: Medicines for Malaria Venture; Pf: Plasmodium falciparum; Pf GLURP: Pf Glutamate-rich protein; Pf MSP-1/2: P. falciparum Merozoite surface protein 1/2; PI: Principal investigator; PMR: Parasite multiplication rate; PRR: Parasite reduction ratio; qPCR: Quantitative Polymerase Chain Reaction; RNA: Ribonucleic acid; SBP: Systolic blood pressure; spp.: Subspecies; ULN: Upper limit of normal; VIS: Volunteer infection studies; WGS: Whole genome sequencing.

\section{Acknowledgements}

These studies were supported by Medicines for Malaria Venture. The authors would like to thank all staff from Q-Pharm Pty Ltd, particularly Paul Morgan, Joan Stark and Miranda Goodwin; Jeremy Gower, Hayley Mitchell and Jenny Peters from QIMR Berghofer in preparing the inoculum; Allan Saul for providing raw data from the original 1995 QIMR study; G. Dennis Shanks from the Australian Defence Force Malaria and Infectious Diseases Institute for his role as the independent medical monitor; staff at the Australian Defence Force Malaria and Infectious Diseases Institute for conducting the in vitro drug sensitivity testing; staff at the Queensland Paediatric Infectious Diseases Laboratory for performing QPCR analysis; Lifeblood for providing human erythrocytes for the MCB production; Dr Maria Rebelo, now works at the University of Lisbon. The authors would also like to thank the volunteers who participated in the studies. 


\section{Authors' contributions}

SDW, MR, MF, RW, KT, JSM and BEB contributed to the design of the study. SDW, MR, MF, SL, LM, FA, HJ, SC, JJM, JSM and BEB participated in the implementation of the study and/or analysis of results. SDW, MR, MF, SL, KT JSM and BEB participated in writing the manuscript. All authors read and approved the final manuscript.

\section{Funding}

QIMR Berghofer Medical Research Institute sponsored the studies, with support from Medicines for Malaria Venture, through funds provided by the Bill and Melinda Gates Foundation (Grant OPP1111147). JSM received funding from a National Health and Medical Research Council of Australia (NHMRC) program Grant (1132975). JSM is also supported by a NHMRC Practitioner Fellowship.

\section{Availability of data and materials}

The datasets used and/or analysed during the current study are available from the corresponding author on reasonable request.

\section{Ethics approval and consent to participate}

Ethical approval was given by QIMR Berghofer Human Research Ethics Committee (HREC), with further ethical approval given by Lifeblood (formerly Australian Red Cross Blood Service) HREC for P. falciparum 3D7 MBE-008. All participants gave written informed consent before enrolment.

\section{Consent for publication}

Not applicable.

\section{Competing interests}

The authors declare that they have no competing interests.

\section{Author details}

${ }^{1}$ QIMR Berghofer Medical Research Institute, Brisbane, QLD, Australia. ${ }^{2}$ Centre for Defence Pathology, Royal Centre for Defence Medicine, Joint Hospital Group, ICT Building, Birmingham Research Park, Vincent Drive, Birmingham, UK. ${ }^{3}$ Clinical Sciences Department, Liverpool School of Tropical Medicine, Pembroke Place, Liverpool, UK. ${ }^{4}$ School of Medicine, University of Queensland, Herston, QLD, Australia. ${ }^{5}$ Medicines for Malaria Venture, 20 Route de Pre-Bois, PO Box 1826, 1215 Geneva 15, Switzerland.

Received: 9 October 2020 Accepted: 5 February 2021

Published online: 16 February 2021

\section{References}

1. Menard D, Dondorp A. Antimalarial drug resistance: a threat to malaria elimination. Cold Spring Harb Perspect Med. 2017;7:a025619.

2. Sinha S, Medhi B, Sehgal R. Challenges of drug-resistant malaria. Parasite. 2014;21:61.

3. WHO. Artemisinin and artemisinin-based combination therapy resistance. Status report. Geneva, World Health Organization, Global Malaria Programme, 2016.

4. Rabinovich RN, Drakeley C, Djimde AA, Hall BF, Hay SI, Hemingway J, et al. malERA: an updated research agenda for malaria elimination and eradication. PLoS Med. 2017;14:e1002456.

5. Engwerda CR, Minigo G, Amante FH, McCarthy JS. Experimentally induced blood stage malaria infection as a tool for clinical research. Trends Parasitol. 2012;28:515-21.

6. Stanisic DI, Mccarthy JS, Good MF. Controlled human malaria infection: applications, advances, and challenges. Infect Immun. 2018;86:e00479-e517.

7. van Huijsduijnen HR, Wells TN. The antimalarial pipeline. Curr Opin Pharmacol. 2018;42:1-6.

8. Wells TN, van Huijsduijnen HR, Van Voorhis WC. Malaria medicines: a glass half full? Nat Rev Drug Discov. 2015;14:424-42.

9. McCarthy JS, Ruckle T, Djeriou E, Cantalloube C, Ter-Minassian D, Baker $M$, et al. A Phase II pilot trial to evaluate safety and efficacy of ferroquine against early Plasmodium falciparum in an induced blood-stage malaria infection study. Malar J. 2016;15:469.
10. McCarthy JS, Baker M, O'Rourke P, Marquart L, Griffin P, van Huijsduijnen $H R$, et al. Efficacy of OZ439 (artefenomel) against early Plasmodium falciparum blood-stage malaria infection in healthy volunteers. J Antimicrob Chemother. 2016;71:2620-7.

11. Woodford J, Collins KA, Odedra A, Wang C, Kyung Jang I, Domingo GJ, et al. An experimental human blood-stage model for studying Plasmodium malariae infection. J Infect Dis. 2020;221:948-55.

12. Alkema M, Reuling IJ, de Jong GM, Lanke K, Coffeng LE, van Gemert G-J, et al. A randomized clinical trial to compare Plasmodium falciparum gametocytemia and infectivity after blood-stage or mosquito biteinduced controlled malaria infection. J Infect Dis; 2020; jiaa157.

13. NCT02927145. A challenge study to assess the safety, immunogenicity and efficacy of a malaria vaccine candidate. ClinicalTrials.gov. https://clin caltrials.gov/ct2/show/. Accessed 28 September 2020.

14. ANZCTR Trial ID: NCT03542149. Phase $1 \mathrm{~b}$ to assess safety, tolerability, pharmacokinetic profile, and antimalarial activity of single doses of co-administered OZ439and PQP against early Plasmodium falciparum blood stage infection in healthy adult volunteers. 2018. http://www.anzct r.org.au/Trial/Registration/TrialReview.aspx?id=7116\&isClinicalTrial=True. Accessed 28 September 2020

15. ANZCTR Trial ID: ACTRN12619001215112. Open label study using the $P$. falciparum induced blood stage malaria (IBSM) model to determine the safety and tolerablity and to characterise the antimalarial activity of a single-dose oral administration of ZY-19489. 2019. https://anzctr.org.au/ Trial/Registration/TrialReview.aspx?ACTRN=12619001215112. Accessed 28 September 2020.

16. Gaur AH, McCarthy JS, Panetta JC, Dallas RH, Woodford J, Tang L, et al. Safety, tolerability, pharmacokinetics, and antimalarial efficacy of a novel Plasmodium falciparum ATP4 inhibitor SJ733: a first-in-human and induced blood-stage malaria phase $1 \mathrm{a} / \mathrm{b}$ trial. Lancet Infect Dis. 2020;20:964-75.

17. Pawliw R, Farrow R, Sekuloski S, Jennings H, Healer J, Phuong T, et al. A bioreactor system for the manufacture of a genetically modified Plasmodium falciparum blood stage malaria cell bank for use in a clinical trial. Malar J. 2018;17:283.

18. Pombo DJ, Lawrence G, Hirunpetcharat C, Rzepczyk C, Bryden M, Cloonan $\mathrm{N}$, et al. Immunity to malaria after administration of ultra-low doses of red cells infected with Plasmodium falciparum. Lancet. 2002;360:610-7.

19. Cheng Q, Lawrence G, Reed C, Stowers A, Ranford-cartwright L, Creasey A, et al. Measurement of Plasmodium falciparum growth rates in vivo: a test of malaria vaccines. Am J Trop Med Hyg. 1997;57:495-500.

20. Watts RE, Odedra A, Marquart L, Webb L, Abd-Rahman AN, Cascales L, et al. Safety and parasite clearance of artemisinin-resistant Plasmodium falciparum infection: a pilot and a randomised volunteer infection study in Australia. PLoS Med. 2020;17:e1003203.

21. ANZCTR Trial ID: ACTRN12617000824369. Safety and immunogeneticity study of a genetically attenuated malaria vaccine candidate in healty volunteers. 2017. https://www.anzctr.org.au/Trial/Registration/TrialRevie w.aspx?id=372949. Accessed 28 September 2020.

22. PIC/S guide to Good Manufacturing Practice for medicinal products Annex 13-Manufacture of investigational medicinal products. The Australian code of Good Manufacturing Practice (GMP) for blood and blood components, human tissues and human cellular therapy products. https://www.tga.gov.au/publication/pe009-13-pics-guide-gmp-medic inal-products. Accessed 28 September 2020.

23. British Pharmacopoeia Commission. British Pharmacopoeia 2016. London: TSO; 2016.

24. Rockett RJ, Tozer SJ, Peatey C, Bialasiewicz S, Whiley DM, Nissen MD, et al. A real-time, quantitative PCR method using hydrolysis probes for the monitoring of Plasmodium falciparum load in experimentally infected human volunteers. Malar J. 2011;10:48.

25. Wockner LF, Hoffmann I, Webb L, Mordmüller B, Murphy SC, Kublin $J G$, et al. Growth rate of Plasmodium falciparum: analysis of parasite growth data from malaria volunteer infection studies. J Infect Dis. 2020;221:963-72

26. Analysing effect sizes. Fixed-effects models. In: Cooper H, Hedges LV, Valentine JC, editors. Handbook of research synthesis and meta-analysis. 2nd ed. New York: Russell Sage Foundation; 2009. p. 279-94.

27. Marquart L, Baker M, O'Rourke P, McCarthy JS. Evaluating the pharmacodynamic effect of antimalarial drugs in clinical trials by quantitative PCR. Antimicrob Agents Chemother. 2015;59:4249-59. 
28. Stein DS, Jain JP, Kangas M, Lefevre G, Machineni S, Griffin P, et al. Openlabel, single-dose, parallel-group study in healthy volunteers to determine the drug-drug interaction potential between KAE609 (cipargamin) and piperaquine. Antimicrob Agents Chemother. 2015;59:3493-500.

29. Woodford J, Shanks GD, Griffin P, Chalon S, McCarthy JS. The dynamics of liver function test abnormalities after malaria infection: a retrospective observational study. Am J Trop Med Hyg. 2018:98:1113-9.

30. Odedra A, Webb L, Marquart L, Britton LJ, Chalon S, Moehrle JJ, et al. Liver Function Test abnormalities in experimental and clinical Plasmodium vivax Infection. Am J Trop Med Hyg. 2020;103:1910-7.

31. Chughlay MF, Akakpo S, Odedra A, Csermak-Renner K, Djeriou E, Winnips C, et al. Liver Enzyme elevations in Plasmodium falciparum volunteer infection studies: findings and recommendations. Am J Trop Med Hyg. 2020;103:378-93.

32. Reuling IJ, de Jong GM, Yap XZ, Asghar M, Walk J, van de Schans LA, et al Liver Injury in uncomplicated malaria is an overlooked phenomenon: an observational study. EBioMedicine. 2018;36:131-9.

33. Moore BR. Liver injury in uncomplicated malaria: an overlooked phenomenon. EBioMedicine. 2018;37:15-6.

34. van Wolfswinkel ME, Langenberg MCC, Wammes LJ, Sauerwein RW, Koelewijn R, Hermsen CC, et al. Changes in total and differential leukocyte counts during the clinically silent liver phase in a controlled human malaria infection in malaria-naïve Dutch volunteers. Malar J. 2017;16:457.

35. Hviid L, Kemp K. What is the cause of lymphopenia in malaria? Infect Immun. 2000;68:6087-9.

36. Brickley EB, Coulibaly M, Gabriel EE, Healy SA, Hume JC, Sagara I, et al. Utilizing direct skin feeding assays for development of vaccines that interrupt malaria transmission: a systematic review of methods and case study. Vaccine. 2016;34:5863-70

37. McCarthy JS, Griffin PM, Sekuloski S, Bright AT, Rockett R, Looke D, et al. Experimentally induced blood-stage Plasmodium vivax infection in healthy volunteers. J Infect Dis. 2013;208:1688-94.

38. Payne RO, Griffin PM, McCarthy JS, Draper SJ. Plasmodium vivax controlled human malaria infection_-Progress and prospects. Trends Parasitol. 2017:33:141-50.

\section{Publisher's Note}

Springer Nature remains neutral with regard to jurisdictional claims in published maps and institutional affiliations.
Ready to submit your research? Choose BMC and benefit from:

- fast, convenient online submission

- thorough peer review by experienced researchers in your field

- rapid publication on acceptance

- support for research data, including large and complex data types

- gold Open Access which fosters wider collaboration and increased citations

- maximum visibility for your research: over $100 \mathrm{M}$ website views per year

At BMC, research is always in progress.

Learn more biomedcentral.com/submissions 